\title{
50GHz Ge Waveguide Electro-Absorption Modulator Integrated in a 220nm SOI Photonics Platform
}

\author{
S. Gupta ${ }^{1,3}$, S. A. Srinivasan ${ }^{1,2}$, M. Pantouvaki ${ }^{1}$, H. Chen ${ }^{1,2}$, P. Verheyen ${ }^{1}$, G. Lepage ${ }^{1}$, D. Van Thourhout ${ }^{2}$ \\ G. Roelkens' ${ }^{2}$ K. Saraswat ${ }^{3}$, P. Absil ${ }^{1}$, J. Van Campenhout ${ }^{1}$ \\ ${ }^{1}$ Imec, Kapeldreef 75, Leuven B-3001, Belgium \\ ${ }^{2}$ Photonics Research Group, Dept. of Information Technology, Ghent University - Imec, St. Pietersnieuwstraat 41, 9000 Ghent, Belgium \\ ${ }^{3}$ Department of Electrical Engineering, Stanford University, Stanford, California 94305, United States \\ Author e-mail address: jvcampen@imec.be
}

\begin{abstract}
We report waveguide-integrated Ge electro-absorption modulators operating at $1615 \mathrm{~nm}$ wavelength with $3 \mathrm{~dB}$ bandwidth beyond $50 \mathrm{GHz}$ and a capacitance of $10 \mathrm{fF}$. A $2 \mathrm{~V}$ voltage swing enables 4.6dB DC extinction ratio for $4.1 \mathrm{~dB}$ insertion loss.

OCIS codes: (250.7360) Waveguide modulators; (230.2090) Electro-optical devices; (200.4650) Optical interconnects.
\end{abstract}

\section{Introduction}

Silicon photonics $(\mathrm{SiPh})$ is considered to be a key enabling technology for future CMOS systems since it has the potential to alleviate the bandwidth-power-density bottleneck of electrical interconnects [1]. However, aggressive energy targets for interconnects have to be met before the technology can become mainstream replacement for their electrical counterparts [1]. In addition to stringent energy and footprint limitations, the optical network should have high bandwidth to support the ever increasing data needs of the end user. In this paper, we report compact Ge electro-absorption modulators (EAM) based on Franz-Keldysh effect [2] with a modulation speed of over 50GHz and a capacitance of 10fF. The Ge EAM devices are integrated in SiPh platform on 200mm silicon-on-insulator (SOI) wafers with $220 \mathrm{~nm}$ top Si thickness, along with various passive and active Si devices. Compared to earlier results on GeSi electro-absorption modulators using a $3 \mu \mathrm{m} \mathrm{SiPh}$ platform [3], the devices in this work have improved performance including higher modulation speed, higher modulation efficiency and lower capacitance due to strong confinement of optical and electrical field enabled by submicron $\mathrm{Ge} / \mathrm{Si}$ waveguide platform.

\section{Design and Fabrication of the Modulator}

The working principle behind bulk Ge electro-absorption modulators is the Franz-Keldysh (FK) effect according to which an applied electric field causes band-tilting thereby increasing the absorption coefficient in the weakly absorbing regime [2]. A significant change in Ge absorption coefficient can be achieved if the electric field difference between OFF and ON state $(\Delta \mathrm{E})$ is greater than $10 \mathrm{kV} / \mathrm{cm}$ as shown in Fig.1(a). A reverse biased p-i-n diode is an obvious choice for realizing such a strong electric field contrast. The dimensions, doping profile and contacting scheme play a crucial role in the overall performance of the modulator. Fig. 1(b) shows a cross-sectional schematic of the Ge EAM devices reported in this paper. A lateral p-i-n diode with moderate doping concentrations $\left(\sim 5 \times 10^{17} \mathrm{~cm}^{-3}\right)$ is implemented in a Ge waveguide. Both the anode and cathode are contacted from highly doped $\mathrm{Si}$ layers $\left(\sim 4 \times 10^{20} \mathrm{~cm}^{-3}\right)$, avoiding the need for direct metal contacts on the Ge waveguide, which would otherwise cause excessive optical absorption loss. Extensive simulations were carried out in Sentaurus ${ }^{\mathrm{TM}}$ to optimize implant conditions for obtaining the strongest optical-electrical interaction. Fig. 1(c) and (d) depict the optical mode and electrical field contrast $\Delta \mathrm{E}$ respectively in the optimized device, showing a strong overlap between the two.
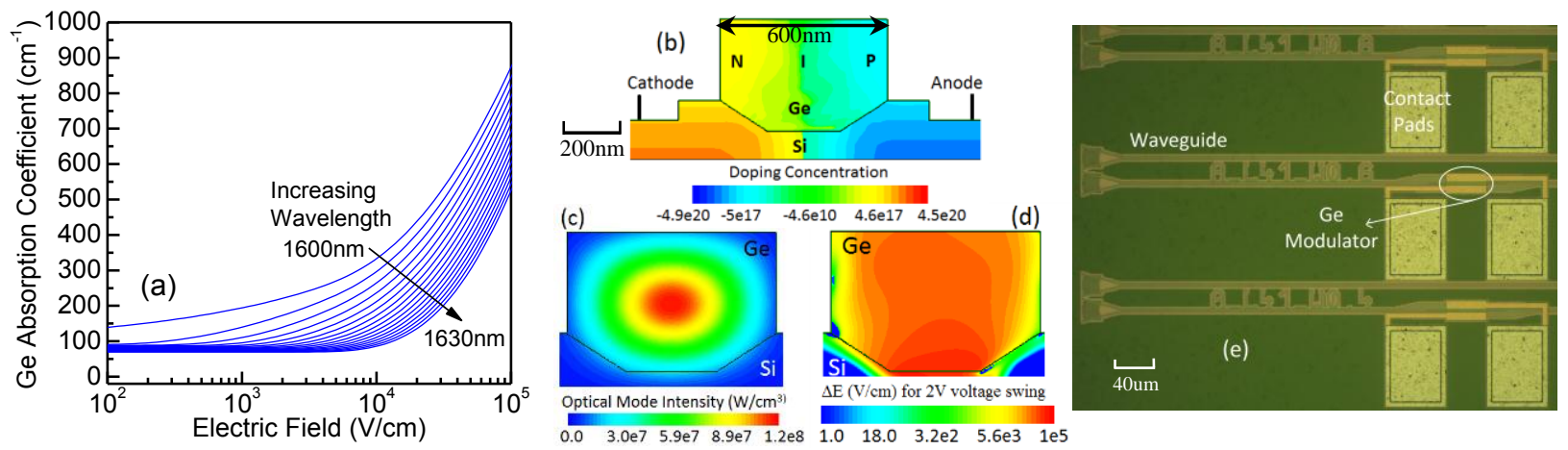

Fig. 1 (a) Modeled absorption coefficient vs. applied electric field. (b) Schematic of the Ge EAM p-i-n diode. (c) Approximated optical field distribution showing good confinement in Ge. (d) Change in electric field ( $\Delta \mathrm{E}$ ) between ON and OFF state. (e) Microscope image of the fabricated modulators integrated with $\mathrm{Si}$ waveguides and grating couplers. 
A set of modulators with varying Ge length (from $10 \mu \mathrm{m}$ to $80 \mu \mathrm{m}$ ) and width (from $400 \mathrm{~nm}$ to $1 \mu \mathrm{m}$ ) were fabricated in imec's $200 \mathrm{~mm}$ Si photonics platform [4]. The Ge thickness was 400nm and the top Si thickness of the SOI wafer was $220 \mathrm{~nm}$. A poly-Si taper structure was implemented to ensure low-loss coupling from the Si waveguide into the Ge waveguide and vice versa. TE mode grating couplers with peak-coupling wavelength around $1600 \mathrm{~nm}$ were used for fiber-chip coupling. Fig. 1(e) shows a top-down microscope image of the Ge modulator EAM array, integrated with Si waveguides.

\section{Modulator Performance}

(a) Static Characteristics: Fig. 2(a) shows I-V characteristics of fabricated EAM devices with varying length. The low dark current of the Ge/Si diodes ( 10nA at $-1 \mathrm{~V}$ for a $40 \mu \mathrm{m}$ long device) signify high quality Ge and controlled electrostatics thereby ensuring very low static energy consumption in the modulator. Fig. 2(b) shows the measured and simulated absorption spectrum with different bias voltages applied to the Ge modulator. The measured change in absorption with bias is clearly evident and is reasonably well matched with theoretical predictions from FK theory and simulation. The absorption spectrum for different bias voltages intersect at $\sim 1580 \mathrm{~nm}$ which corresponds to the $\mathrm{HH}-\Gamma$ band gap for epitaxial Ge on Si. Fig. 2(c) shows the static extinction ratio and insertion loss for a $40 \mu \mathrm{m}$ long and $600 \mathrm{~nm}$ wide device. For a $2 \mathrm{~V}$ swing at $1615 \mathrm{~nm}$, the insertion loss (IL) is $4.14 \mathrm{~dB}$ and the extinction ratio (ER) is $4.58 \mathrm{~dB}$. It should be noted that all the optical data is normalized to a neighboring straight waveguide, but it includes the coupling loss between the Si waveguide and the Ge modulator.
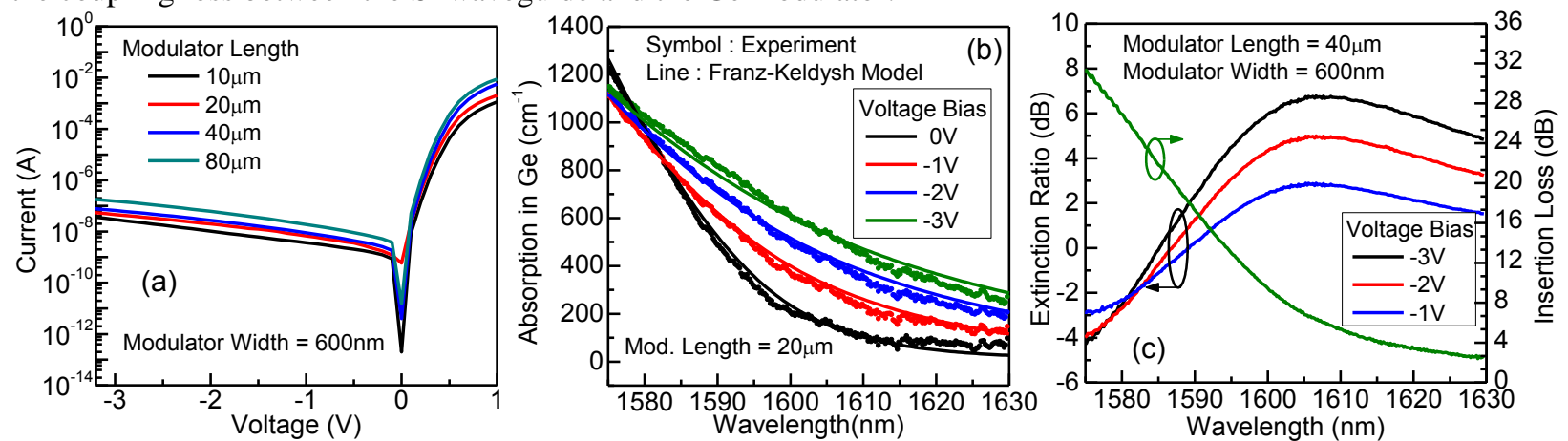

Fig. 2 (a) Dark I-V of modulators with varying length. (b) measured change in absorption coefficient of Ge is well matched by simulations. (c) extinction ratio and insertion loss of a representative device.

An additional benchmark, comparing the ratio between ER and IL, is shown in figure 3(a). It is clear that the modulator of $40 \mu \mathrm{m}$ length and $600 \mathrm{~nm}$ width has the best performance. Longer devices suffer of increased IL while shorter devices don't exhibit sufficient ER. Similarly, wider devices suffer from lower $\Delta \mathrm{E}$ while narrower devices have excessive coupling loss. Another important figure of merit is the Link Power Penalty (LPP) defined as ( $\mathrm{P}_{\text {out }}(1)$ $\left.\mathrm{P}_{\text {out }}(0)\right) /\left(2 \times \mathrm{P}_{\text {in }}\right)$, where $\mathrm{P}_{\text {out }}(1)$ and $\mathrm{P}_{\text {out }}(0)$ are the high and low level of output optical power and $\mathrm{P}_{\text {in }}$ is the input optical power [3]. Fig. 3(b) shows that the minimum LPP for the best device is $8.2 \mathrm{~dB}$ for $2 \mathrm{~V}$ swing. The usable optical bandwidth is defined as the wavelength range between which the LPP remains within $1 \mathrm{~dB}$ of its minimum value. Due to the limitations in available laser wavelengths, we could measure only half of this bandwidth which came out to be $17.5 \mathrm{~nm}(1612.5-1630 \mathrm{~nm})$ for $2 \mathrm{~V}$ swing. Based on simulations, we can extrapolate the full $1 \mathrm{~dB}$ optical bandwidth to be greater than 35nm. Fig. 3(c) shows the variation of minimum LPP with modulator length and voltage swing. The trends for LPP are very similar to that of ER/IL seen before.
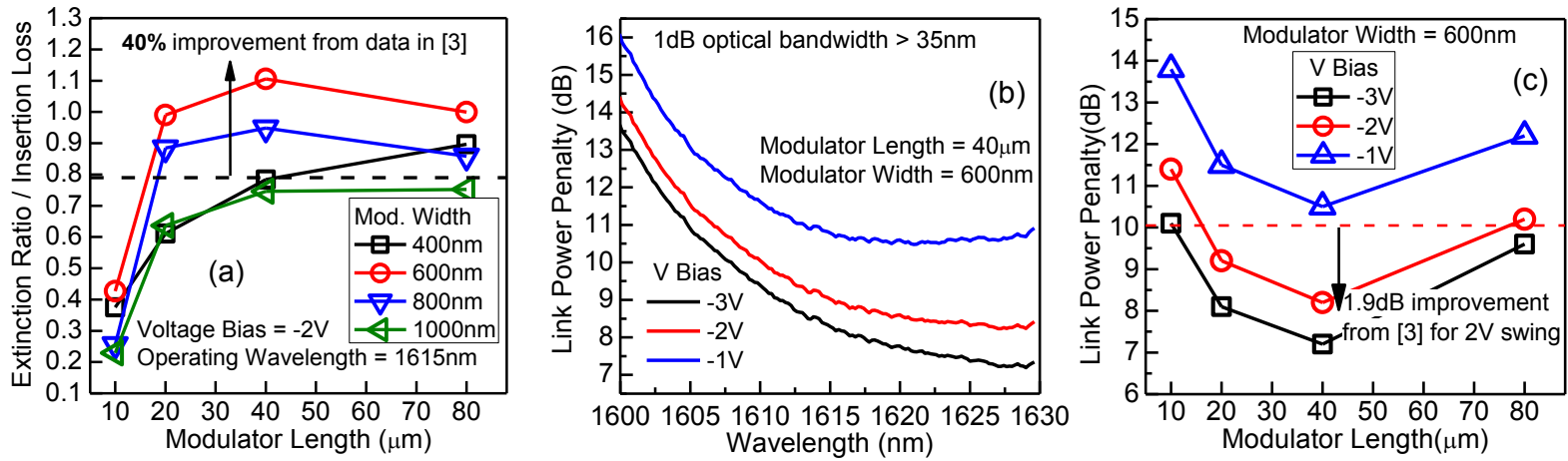

Fig. 3 (a) Figure of merit (ER/IL) versus modulator length and width. (b) Link Power Penalty (LPP) of the best device. (c) Variation of minimum LPP with modulator length and voltage swing. 

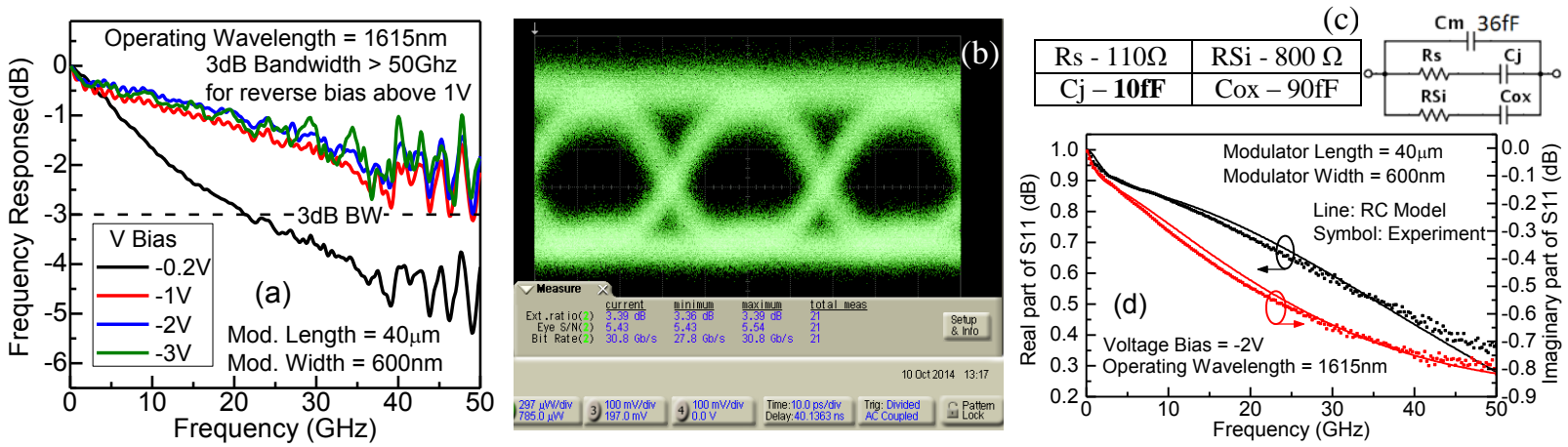

Fig. 4 (a) RF S21 measurements showing $>50 \mathrm{GHz}$ bandwidth. (b) Eye diagram showing clear opening at 28Gbps with a dynamic ER of $3.39 \mathrm{~dB}$ for a nominal 2Vpp swing. (c) Equivalent Circuit and (d) $\mathrm{S} 11$ parameter fit showing $\mathrm{Cj}=10 \mathrm{fF}$ and $\mathrm{Rs}=110 \Omega$.

(b) High-Speed Performance: The 3dB bandwidth (BW) of the modulator is greater than $50 \mathrm{GHz}$ for reverse bias above $1 \mathrm{~V}$, as extracted from electro-optic S21 measurements shown in Fig. 4(a). The FK effect is known to be a sub-picosecond effect [5] and therefore the speed of the EAM modulators is expected to be RC limited. Fig. 4(b) shows the measured eye diagram at $1610 \mathrm{~nm}$ for a $28 \mathrm{Gbps}$ data stream (PRBS31) generated by a pattern generator and delivered to the device by a $50 \Omega$ terminated RF probe. An open eye diagram with dynamic ER of $3.39 \mathrm{~dB}$ at $2 \mathrm{~V}$ swing can be observed. Since the BW of the modulator is more than $50 \mathrm{GHz}$, one can expect it to operate at data rates as high as 56Gbps. The RC model of the EAM can be extracted by fitting the $\mathrm{S} 11$ parameters of the equivalent circuit [6] shown in Fig. 4(c). A reasonable fit yields a junction capacitance of $10 \mathrm{fF}$ and series resistance of $110 \Omega$ for $2 \mathrm{~V}$ reverse bias, as shown in Fig. 4(d). This translates to an estimated dynamic energy dissipation of just 10fJ/bit for a $2 \mathrm{~V}$ swing. The relatively small height and length of the Ge modulator leads to a low junction capacitance and hence better high speed performance with minimal energy consumption. For benchmarking this work, Table 1 summarizes the key figures of merit with other representative modulators, illustrating the relative advantages of the presented Ge EAM versus other Si-based modulator types and earlier $\mathrm{Ge}(\mathrm{Si}) \mathrm{EAM}$ demonstrations.

\section{Conclusion}

We have demonstrated a CMOS compatible, waveguide integrated Ge EAM with a bandwidth greater than 50GHz, capacitance of $10 \mathrm{fF}$ and link power penalty of $8.2 \mathrm{~dB}$ for a $2 \mathrm{Vpp}$ drive swing. The demonstrated device shows good potential for enabling power-efficient and compact $28 \mathrm{G}, 40 \mathrm{G}$ and $56 \mathrm{G}$ electro-optical transmitters.

This work was supported by imec's industry-affiliation program on Optical I/O. The authors acknowledge imec's 200mm CMOS line for device fabrication and imec's PDK team for mask data preparation and tape out. Device layout was performed in IPKISS provided by Luceda Photonics.

Table 1: Performance metrics for different representative modulators reported experimentally

\begin{tabular}{|c|c|c|c|c|c|c|c|}
\hline $\begin{array}{c}\text { Modulator } \\
\text { Type }\end{array}$ & $\begin{array}{c}3 \mathrm{~dB} \text { BW } \\
(\mathrm{GHz})\end{array}$ & $\begin{array}{c}\text { Modulator } \\
\text { Capacitance }(\mathrm{fF})\end{array}$ & $\begin{array}{c}\text { V Swing } \\
(\mathrm{V})\end{array}$ & $\begin{array}{c}\text { Active } \\
\text { Footprint }\left(\mu \mathrm{m}^{2}\right)\end{array}$ & $\begin{array}{c}\text { ER } \\
(\mathrm{dB})\end{array}$ & $\begin{array}{c}\text { IL } \\
(\mathrm{dB})\end{array}$ & $\begin{array}{c}\text { Optical } \\
\text { BW }(\mathrm{nm})\end{array}$ \\
\hline This work & $\mathbf{5 0}$ & $\mathbf{1 0}$ & $\mathbf{2}$ & $\mathbf{2 4}$ & $\mathbf{4 . 6}$ & $\mathbf{4 . 1}$ & $>\mathbf{3 5}$ \\
\hline GeSi FK [3] & 34 & 69.6 & 2 & 40 & 3.8 & 4.8 & 40 \\
\hline GeSi FK [7] & 1.2 & 11 & 3 & 30 & 8 & 3.7 & 14 \\
\hline GeSi QCSE* $^{*}[8]$ & 3.5 & 3 & 1 & 8 & 3.2 & 15 & 20 \\
\hline GeSi QCSE* $^{*}[9]$ & 6.3 & 200 & 3 & 400 & 4 & 3 & 20 \\
\hline Si MZI* $^{*}[10]$ & 30 & $\sim 800$ & 1.5 & $\sim 1500$ & 3.4 & 7.1 & $>80$ \\
\hline Si Ring [11] & 21 & $\sim 17$ & 0.5 & 20 & 6.4 & 1.2 & $<0.1$ \\
\hline
\end{tabular}

*QCSE: Quantum Confined Stark Effect; **MZI: Mach-Zehnder interferometer

[1] D.A.B. Miller, "Device Requirements for Optical Interconnects to Silicon Chips", Proc. IEEE, vol. 97, no. 7, pp. 1166-1185, Jul. 2009.

[2] D.A.B. Miller, et al. "Relation between electroabsorption in bulk semiconductors and in quantum wells: The quantum-confined FranzKeldysh effect", Phys. Rev. B 33, 6976 (1986)

[3] D. Feng, et al. "High-speed GeSi electro-absorption modulator on the SOI waveguide platform," IEEE JSTQE, 19(6), 3401710 (2013)

[4] P. Verheyen, et al. "Highly Uniform $25 \mathrm{~Gb} / \mathrm{s} \mathrm{Si}$ Photonics Platform for High-Density, Low-Power WDM Optical Interconnects," in Advanced Photonics for Communications, OSA Technical Digest, 2014, paper IW3A.4.

[5] J.F. Lampin, et al. "Detection of picosecond electrical pulses using the intrinsic Franz-Keldysh effect", APL 78, 4103-4105 (2001)

[6] G. Li, et al. "Ultralow-power, high- performance Si photonic transmitter," OFC 2010, SanDiego, CA, USA, 2010, paper no. OMI2

[7] J. F. Liu, et al. "Waveguide- integrated, ultralow-energy GeSi electro-absorption modulators", Nat. Photonics 2(7), $433-437$ (2008)

[8] S. Ren, et al. "Ge/SiGe quantum well waveguide modulator monolithically integrated with SOI waveguides," IEEE Photon. Technol. Lett.,

vol. 24 , no. 6, pp. 461-463, Mar. 2012

[9] P. Chaisakul, et al. "Integrated germanium optical interconnects on silicon substrates", Nature Photonics 8, 482- 488 (2014)

[10] M. Streshinsky, et al., "Low power $50 \mathrm{~Gb} / \mathrm{s}$ silicon traveling wave Mach-Zehnder modulator near 1300 nm," Opt. Express 21, (2013)

[11] E. Timurdogan, et al., "An ultralow power athermal silicon modulator," Nat. Commun. 5, (2014) 